\title{
Proteínas del gluten y reología de trigos harineros mexicanos influeciados por factores ambientales y genotípicos
}

\author{
Micaela De la O Olán(1), Eduardo Espitia Rangel(2), Héctor Eduardo Villaseñor Mir(2), José Domingo Molina Galán(1), \\ Higinio López Sánchez ${ }^{(3)}$, Amalio Santacruz Varela(1), y Roberto Javier Peña Bautista ${ }^{(4)}$
}

\begin{abstract}
(1)Colegio de Postgraduados (Colpos), Programa de Recursos Genéticos y Productividad, Km 36,5 Carretera México-Texcoco, Código Postal 56230, Montecillo, Estado de México, México. E-mail: micad@colpos.mx, jmolina@colpos.mx, asvarela@colpos.mx ${ }^{(2)}$ Instituto Nacional de Investigaciones Forestales, Agrícolas y Pecuarias, Programa de Trigo, Campo Experimental Valle de México, Código Postal 56230, Chapingo, Estado de México, México. E-mail: espitia.eduardo@inifap.gob.mx, hevimir3@yahoo.com.mx ${ }^{(3)}$ Colpos, Campus Puebla, Km 125,5, Carretera Federal México-Puebla, Santiago, Momoxpan, Código Postal 72720 San Pedro Cholula, Puebla, México. E-mail: higiniols@colpos.mx (4)Centro Internacional de Mejoramiento de Maíz y Trigo, Programa de Trigo, Apartado Postal 6-641, Código Postal 06600 Distrito Federal, México. E-mail: j.pena@cgiar.org
\end{abstract}

Resumen - El objetivo de este estudio fue conocer el efecto de factores ambientales y genotípicos sobre los parámetros de calidad industrial y sobre la cantidad y relación de proteínas monoméricas y poliméricas del gluten en 24 líneas recombinantes de trigos harineros de temporal. El cultivo se desarrolló en cinco condiciones ambientales generadas por manejo agronómico, ciclo otoño-invierno 2006/2007, en Roque, Guanajuato, México. Se evaluaron el tiempo de amasado (TMA), fuerza (ALVW), extensibilidad (ALVPL) de la masa, fracción rica en gliadina (50PS) y en glutenina (50PI), y su relación (50PS/50PI). Las mejores combinaciones de gluteninas de alto y bajo peso molecular para TMA y ALVW fueron los genotipos con 1, 17+18, 5+10/Glu-A3c, Glu-B3g, Glu-D3b; 1, 17+18, 5+10/Glu-A3c, Glu-B3h, Glu-D3b, y 2*, 17+18, 5+10/Glu-A3c, Glu-B3g, Glu-D3b; para ALVPL, 2*, 17+18, 2+12/Glu-A3e, Glu-B3h, Glu-D3b; para 50PS, 2*, 17+18, 2+12/Glu-A3e, Glu-B3h, Glu-D3b; y 1, 17+18, 5+10/Glu-A3e, Glu-B3h, Glu-D3b. La relación 50PS/50PI fue mayor en genotipos con 2*, 17+18, 2+12/Glu-A3e, Glu-B3g, Glu-D3b. El TMA es mayor cuando aumenta la temperatura y la mejor ALVPL se obtiene en el ambiente bajo condiciones normales. La fracción 50PS y la relación 50PS/50PI son mayores cuando se realiza la fertilización con azufre, y se obtiene incremento de 50PI con riego limitado y aumento de temperaturas durante el llenado de grano.

Términos para indexación: Triticum aestivum, calidad industrial, contenido de proteína, gliadina, glutenina.

\section{Gluten proteins and rheology of Mexican bread wheats as affected by environmental and genotypic factors}

\begin{abstract}
The objective of this study was to determine the effect of environmental and genotypic factors on industrial quality and on the monomeric and polymeric protein contents and ratio of 24 rainfed bread wheat recombinant lines. The cultivation was done in five environmental conditions generated by agronomic management in the autumn-winter 2006/2007 cycle, at Roque, Guanajuato, Mexico. The mixing time (TMA), strength (ALVW) and extensibility (ALVPL) of the dough, gliadin (50PS) and glutenin (50PI) rich fractions, and their ratio (50PS/50PI), were evaluated. The best combinations of high and low molecular weight glutenins for TMA and ALVW were 1, 17+18, 5+10/Glu-A3c, Glu-B3g, Glu-D3b; 1, 17+18, 5+10/Glu-A3c, Glu-B3h, Glu-D3b; and 2*, 17+18, 5+10/Glu-A3c, Glu-B3g, Glu-D3b; for ALVPL it was 2*, 17+18, 2+12/Glu-A3e, Glu-B3h, Glu-D3b; and for 50PS they were 2*, 17+18, 2+12/Glu-A3e, Glu-B3h, Glu-D3b; and 1, 17+18, 5+10/ Glu-A3e, Glu-B3h, Glu-D3b. The 50PS/50PI ratio was higher in genotypes with 2*, 17+18, 2+12/Glu-A3e, $G l u-B 3 g, G l u-D 3 b$. TMA is higher when temperature increases, and the best ALVPL is found under normal environmental conditions. Gliadin fraction and the 50PS/50PI ratio are higher when sulphur fertilization is done, and 50PI increases with drought and higher temperatures during grain-filling stage.
\end{abstract}

Index terms: Triticum aestivum, industrial quality, protein content, gliadin, glutenin.

\section{Introducción}

La calidad panadera del trigo (Triticum aestivum L.) es un carácter complejo y depende de factores genéticos, efectos ambientales y de manejo agronómico. Son muy pocos los estudios que se han realizado sobre el comportamiento de las variaciones alélicas dentro del genoma de trigo para las fracciones de proteína. Las proteínas son los componentes más importantes del grano de trigo en la definición de la calidad panadera, $\mathrm{y}$ 
las gluteninas y gliadinas son las que forman el gluten, el cual confiere visco-elasticidad a la masa.

Las gliadinas y las gluteninas se encuentran bajo el control de los loci complejos Gli-1/Gli-2 y Glu-1/Glu-3, respectivamente, localizados en los cromosomas 1 y 6 de los genomas A, B y D (Payne et al., 1987). Las gliadinas son monómeros que interactúan por fuerzas no covalentes, y están asociadas al aumento de la viscosidad y extensibilidad de la masa (Uthayakumaran \& Lukow, 2005). Las gluteninas son polímeros compuestos por multicadenas de alto y bajo peso molecular estabilizados por puentes disulfuro, relacionadas con la fuerza, elasticidad y el tiempo del desarrollo de la masa, que pueden separarse en dos grupos con base en el tamaño de los polipéptidos que forman sus polímeros: las subunidades de bajo (G-BPM) y de alto peso molecular (G-APM) (Shewry et al., 2003).

Las fracciones de gluteninas y gliadinas consisten de proteínas de almacenamiento que se acumulan durante el periodo de llenado de grano y son usadas como fuentes de nitrógeno durante la germinación de la semilla (Shewry \& Halford, 2002). La calidad de proteína, definida básicamente por la variedad, es al menos tan importante como la cantidad. Si bien existen diferencias entre distintos genotipos, la cantidad de proteína del grano de trigo está muy influenciada por las condiciones ambientales en que fue cultivado, siendo importante que el trigo tenga suficiente disponibilidad de nitrógeno en el suelo (Shewry et al., 2003).

Diversos estudios de campo indican que las condiciones ambientales, particularmente fertilización y temperatura, afectan la cantidad, composición y polimerización de las proteínas del gluten (Graybosch et al., 1995). Los efectos de la temperatura sobre las proteínas de reserva no son claros y pueden variar con el genotipo (DuPont \& Altenbach, 2003). Altas temperaturas ocasionaron incrementos en la relación gliadinas/gluteninas en la harina de diferentes variedades cultivadas en ambientes controlados (Panozzo \& Eagles, 2000). Cuando las plantas de trigo no son fertilizadas adecuadamente, el contenido de proteína puede mostrar incrementos por efecto de altas temperaturas, debido al efecto diferencial de este factor sobre la deposición de proteína y almidón en grano, y esto puede o no llevar a diferencias en el porcentaje de proteína y calidad de harina (Daniel \& Triboï, 2000).
En México, aunque ya se cuente con genotipos de trigo para la producción de grano, no se conoce la calidad de ellos con base en cantidad, tipos y proporciones de proteínas, particularmente las gluteninas, y cómo estas pueden ser modificadas en los genotipos por el ambiente de producción.

El objetivo de este trabajo fue conocer el efecto de factores ambientales sobre los parámetros de calidad industrial, y especialmente sobre la cantidad y relación de proteínas monoméricas (principalmente gliadinas) y poliméricas (principalmente gluteninas) del gluten en nuevos genotipos (líneas recombinantes) de trigos harineros de temporal.

\section{Materiales y Métodos}

En el presente trabajo, se utilizaron las variedades de trigo harinero Rebeca F2000 y Verano S91 y un grupo de 24 líneas desarrolladas por descendencia de una sola semilla de $\mathrm{F}_{2} \mathrm{a} \mathrm{F}_{7}$, a partir de la cruza entre las dos variedades. Rebeca F2000 posee las subunidades de gluteninas de alto peso molecular 1 en Glu- $A 1,17+18$ en $G l u-B 1$ y $5+10$ en $G l u-D 1$; y de bajo peso molecular "c" en Glu-A3, "g" en Glu-B3 y "b" en Glu-D3; mientras que Verano $S 91$ posee las subunidades de gluteninas de alto peso molecular 2* en Glu-A1, 17+18 en Glu-B1 y $2+12$ en $G l u-D 1$, y las de bajo peso molecular "e" en Glu-A3, "h" en Glu-B3 y "b" en Glu-D3.

Los 26 genotipos se sembraron en el Campo Experimental del Bajío (CEBAJ), del Instituto Nacional de Investigaciones Forestales, Agrícolas y Pecuarias (Inifap), en Roque, Guanajuato, México, en el ciclo otoño-invierno de 2006/2007. El cultivo se desarrolló en cinco condiciones ambientales diferentes, generados mediante manejo agronómico de riego, nitrógeno, azufre, y fecha de siembra tardía, que implica más calor en llenado de grano (Cuadro 1). Se utilizó un diseño experimental de bloques completos al azar con dos repeticiones. La unidad experimental consistió de cuatro surcos de un metro de longitud con una separación de $30 \mathrm{~cm}$ entre ellos. El cultivo se condujo según las recomendaciones del Inifap para el Bajío. La fertilización en todos los ambientes se realizó con la aplicación de la mitad de los fertilizantes al momento de la siembra, y la otra mitad en la etapa de amacollamiento.

Los análisis de calidad se efectuaron en el Laboratorio de Calidad de Trigo, del Centro 
Internacional de Mejoramiento de Maíz y Trigo (CIMMYT). Las características de dureza de grano y contenido de proteína fueron determinadas por medio de espectroscopía (reflectancia) en el rango del infrarojo cercano (NIRS, por su sigla en inglés), con el equipo NIRSystems 6500 (FOSS-Tecator, Hillerød, Denmark). La molienda de grano para generar harina refinada de prueba se efectuó en un molino Brabender Senior. Las propiedades reológicas de las masas fueron determinadas de acuerdo a los métodos de la American Association of Cereal Chemists (AACC International, 1995), en el mixógrafo National con ensayo de absorción variable para determinar el tiempo de amasado, y en el alveógrafo de Chopin, utilizando con absorción variable (50-55\%), con base en la dureza del grano (Peña et al., 1990), para determinar fuerza de masa W (ALVW) y la relación tenacidad/extensibilidad P/L (ALVPL). Las proteínas del gluten fueron fraccionadas por solubilidad en propanol al 50\% en gliadinas y gluteninas, seguida de separación por electroforesis (SDS-PAGE) en alelos de gliadinas y gluteninas, por el procedimiento de Peña et al. (2004). Los alelos de glutenina de alto peso molecular (G-APM) (loci Glu-A1, Glu-B1 y Glu-D1) se identificaron con base en la nomenclatura propuesta por Payne et al. (1987), y las de bajo peso molecular (G-BPM), (loci Glu-A3 y Glu-B3), de acuerdo con Singh et al. (1991), Jackson et al. (1996) y Branlard et al. (2003). En el locus Glu-D3, se usó la nomenclatura de Branlard et al. (2003).

El fraccionamiento y cuantificación de las proteínas se hizo por espectrofotometría, de acuerdo con el protocolo de Suchy et al. (2007). Se colocaron $10 \mathrm{mg}$ de harina refinada (humedad base de $140 \mathrm{~g} \mathrm{~kg}^{-1}$ ) en un tubo Eppendorf de $2 \mathrm{~mL}$; las muestras fueron extraídas con $1.8 \mathrm{~mL}$ de 2-propanol al $50 \%(\mathrm{v} / \mathrm{v})$ durante 30 min, con agitación vigorosa en un Thermomixer Eppendorf (Eppendorf-Netheler, Hamburg, Germany) a $1.100 \mathrm{rpm}$ y $25^{\circ} \mathrm{C}$. Después de la extracción, las muestras se centrifugaron a $12.000 \mathrm{rpm}$ por cinco

Cuadro 1. Tratamientos ambientales que afectaran la calidad de trigos harineros (cruza Rebeca- F2000×Verano-S91).

\begin{tabular}{lcccc}
\hline Tratamiento & Riegos & Fertilización & $\begin{array}{c}\text { Aplicación } \\
\text { de azufre }\end{array}$ & $\begin{array}{c}\text { Fecha } \\
\text { de siembra }\end{array}$ \\
\hline Riego limitado & 3 & $300-60-00$ & no & $7-12-06$ \\
Azufre & 5 & $300-60-00$ & sí & $7-12-06$ \\
Nitrógeno & 5 & $120-60-00$ & no & $7-12-06$ \\
Control & 5 & $300-60-00$ & no & $7-12-06$ \\
Calor & 5 & $300-60-00$ & no & $5-01-07$ \\
\hline
\end{tabular}

min. Los sobrenadantes fueron transferidos a tubos de $5 \mathrm{~mL}$ en duplicados, los cuales fueron colocados en el espectrofotómetro Beckman a $280 \mathrm{~nm}$. Esta fracción, que contiene las proteínas monoméricas y ricas en gliadina, fue llamada 50PS (proteína soluble en propanol). Para el caso de la fracción de proteína soluble total (PST), la cual contiene del 90 al 95\% de las proteínas presentes en la harina, se procedió de la misma manera que para 50PS, excepto que para la extracción, en este caso, se utilizaron $1.8 \mathrm{~mL}$ de ditiotreitol(DTT) además de 2-propanol, y la incubación se realizó a $55^{\circ} \mathrm{C}$. El contenido de proteína insoluble en alcohol, llamada 50PI (rica en glutenina G-APM y G-BPM, o proteína polimérica), en la harina, fue calculada por la diferencia entre el contenido de PST y 50PS. Los resultados de 50PS y 50PI se presentan como proporciones con respecto a PST y se expresan en porcentaje. Adicionalmente, se calculó la relación entre las fracciones ricas en gliadina/ricas en glutenina (50PI/50PS).

Se realizó un análisis de varianza general para las variables estudiadas. Posteriormente, la fuente de variación genotipos se desglosó, y se realizó el análisis de varianza adicional al análisis general, para obtener los cuadrados medios respectivos de combinaciones alélicas o grupos genotípicos Glu-A1, Glu-D1, Glu-A3 y $G l u-B 3$, todo ello mediante el procedimiento GLM de SAS (SAS Institute, 1997). Previamente al análisis de varianza de 50PS y 50PI, se realizó una transformación logarítmica de los datos porcentuales, para corregir la falta de ajuste a una distribución normal de la escala porcentual y no apartarse del supuesto de normalidad que requiere el análisis de varianza. Se realizó una comparación de medias por ambiente, combinaciones obtenidas de la cruza Rebeca F200 x Verano S91 y por cada locus, por la prueba de Tukey al 5\% de probabilidad; además se obtuvieron los coeficientes de correlación de Pearson con el programa SAS (SAS Institute, 1997).

\section{Resultados y Discusión}

En el análisis de varianza general (Cuadro 2), se detectaron diferencias significativas tanto para ambientes como para genotipos en todas las variables de calidad - tiempo de amasado, fuerza y extensibilidad de la masa -, lo que coincide con Souza et al. (2004) y Espitia et al. (2003), quienes observaron 
que las variaciones en características de calidad se deben principalmente a condiciones variables de los ambientes. Para la distribución de las fracciones de proteínas del gluten, así como en la relación entre las fracciones ricas en gliadina y glutenina (50PS/50PI), la fuente de variación ambiente resultó altamente significativa. El manejo de la fertilización nitrogenada, azufrada, el riego limitado y la alta temperatura durante el llenado de grano son factores que influencian la deposición o acumulación de proteínas, lo que coincide con lo reportado por Triboï et al. (2000) y Zhu \& Khan (2001), quienes mencionan que la composición de la proteína en grano depende primeramente del genotipo, pero es significativamente afectada por factores ambientales.

En la interacción genotipo por ambiente, hubo efecto altamente significativo para tiempo de amasado, extensibilidad de la masa, fracción 50PS (rica en gliadina), fracción 50PI (rica en glutenina) y relación 50PS/50PI, y efecto no significativo para fuerza de la masa, lo que concuerda con lo reportado por Souza et al. (2004), quienes concluyen que existen algunos parámetros de calidad que no son afectados por la interacción genotipo-ambiente.

Las combinaciones alélicas resultaron altamente significativas para todas las variables en estudio, lo que indica que los diferentes alelos de gluteninas de alto y bajo peso molecular, de manera conjunta, ejercen efecto diferencial sobre las propiedades viscoelásticas de la masa, en la fracción de las proteínas, y en la relación fracción rica en gliadina/rica en glutenina. El efecto individual de los loci de gluteninas de alto peso molecular, en $G l u-A 1$, fue altamente significativo para tiempo de amasado y fuerza de la masa, y no significativo para extensibilidad de la masa, fracciones 50PS, 50PI y la relación 50PS/50PI. En Glu-D1, ese efecto resultó ser altamente significativo para tiempo de amasado, fuerza y extensibilidad de la masa, 50PS, 50PI, así como la relación 50PS/50PI. Para las gluteninas de bajo peso molecular, el locus Glu-A3 presentó significancia estadística para todas las variables en estudio, excepto para la fracción 50PS y 50PI; el locus Glu-B3 fue altamente significativo para fuerza y extensibilidad de la masa, y no significativo para tiempo de amasado, 50PI, 50PS y relación 50PS/50PI.

Las diferencias significativas, encontradas en los diferentes loci y en sus combinaciones, indican que existe un gran potencial en la manipulación de diferentes parámetros de calidad harinera y aumento en la cantidad de proteínas, lo que enfoca el mejoramiento genético en un número de loci factibles de manejar.

En la prueba de comparación de medias de las combinaciones de gluteninas de alto y bajo peso molecular, se obtuvieron siete combinaciones alélicas, considerándose las de los progenitores (Cuadro 3). Algunas combinaciones superaron en calidad a las combinaciones que poseen los progenitores. Las combinaciones 1, 17+18, 5+10/Glu-A3c, Glu-B3g, Glu-D3b (Rebeca F2000); 1, 17+18, 5+10/Glu-A3c, Glu-B3h, Glu-D3b; y 2*, 17+18, 5+10/Glu-A3c, Glu-B3g, Glu-D3b presentaron los mayores valores de tiempo de amasado, con resultados estadísticamente similares a la combinación $1,17+18,5+10 / G l u-A 3 e$, $G l u-B 3 g$, Glu-D3b, que no difieren de la combinación 1, 17+18, 5+10/Glu-A3e, Glu-B3h, Glu-D3b. Es decir, las combinaciones con la subunidad $5+10$ tienen efecto

Cuadro 2. Cuadrados medios del análisis de varianza de la calidad de trigos harineros (cruza Rebeca F2000 × Verano S91).

\begin{tabular}{|c|c|c|c|c|c|c|c|}
\hline Fuente de variación & GL & MTA & ALVW & ALVPL & 50PS & $50 \mathrm{PI}$ & 50PS/50PI \\
\hline Repeticiones & 1 & $0,001^{\mathrm{ns}}$ & $2441,2^{\text {ns }}$ & $0,029^{\mathrm{ns}}$ & $2,66^{\mathrm{ns}}$ & $2,65^{\mathrm{ns}}$ & $0,053^{\mathrm{ns}}$ \\
\hline Ambientes (A) & 4 & $0,402 * *$ & $13819,5 * *$ & $0,942 * *$ & $583,08 * *$ & $583,11 * *$ & $3,83 * *$ \\
\hline $\mathrm{G} \times \mathrm{A}$ & 86 & $0,202 * *$ & $2571,0 \mathrm{~ns}$ & $0,095 * *$ & $17,93 * *$ & $17,91 * *$ & $0,139 * *$ \\
\hline Genotipos (G) & 25 & $4,3 * *$ & $65553,6^{* *}$ & $0,760 * *$ & $32,78 * *$ & $32,76 * *$ & $0,251 * *$ \\
\hline Combinaciones & 6 & $10,4^{* *}$ & $166473,1^{* *}$ & $1,78 * *$ & $56,23 * *$ & $56,21 * *$ & $0,458 * *$ \\
\hline Locus Glu-A1 & 1 & $17,0 * *$ & $34030,8^{* *}$ & $0,382^{\text {ns }}$ & $23,26^{\mathrm{ns}}$ & $23,20^{\text {ns }}$ & $0,218^{\mathrm{ns}}$ \\
\hline Locus Glu-D1 & 1 & $54,2 * *$ & $996486,5^{* *}$ & $2,1 * *$ & $232,28^{* *}$ & $232,30 * *$ & $1,9^{* *}$ \\
\hline Locus Glu-A3 & 1 & $45,8 * *$ & $475630,9 * *$ & $1,3 * *$ & $83,22^{\mathrm{ns}}$ & $83,21^{\mathrm{ns}}$ & $0,587 *$ \\
\hline Locus Glu-B3 & 2 & $0,594^{\mathrm{ns}}$ & $61742,8^{* *}$ & $2,0 * *$ & $0,06^{\mathrm{ns}}$ & $9,03^{\mathrm{ns}}$ & $0,0332^{\mathrm{ns}}$ \\
\hline Error & 113 & 0,09 & 2313,1 & 0,057 & 307,9 & 302,3 & 0,058 \\
\hline
\end{tabular}

${ }^{n s}$ No significativo. *, **Diferencias significativas al 5 y $1 \%$ de probabilidad, respectivamente. GL, grados de libertad; MTA, tiempo de amasado; ALVW, fuerza de la masa; ALVPL, extensibilidad de la masa; 50PS, fracción rica en gliadina; 50PI, fracción rica en glutenina; 50PS/50PI, relación fracción rica en gliadina/fracción rica en glutenina. 
positivo sobre el tiempo de amasado, lo que concuerda con lo reportado por Radovanovic et al. (2002).

Las combinaciones anteriores presentaron valores altos para alveograma-W, que es una característica asociada con alta fuerza de la masa, lo que es deseable en la industria de la panificación mecanizada. Las combinaciones 2*,17+18, 2+12/Glu-A3e, Glu-B3h, Glu-D3b y 2*, 17+18, 2+12/Glu-A3e, Glu-B3g, GluD3b fueron las de menor fuerza de la masa, aptas para la industria de la panificación manual y semi-mecanizada. En alveograma-P/L, lo ideal es tener una combinación con menores valores, debido a que implican en mayor extensibilidad de la masa, y es por ello que la combinación 2*, 17+18,2+12/Glu-A3e, Glu-B3h, Glu-D3b fue la más adecuada para la calidad panadera.

Por lo general, las combinaciones que poseen el alelo de glutenina de alto peso molecular $2+12$ son las de mayor extensibilidad, y éste es uno de los parámetros más buscados en los genotipos. Para la fracción rica en gliadina (50PS), los valores más altos se observaron con las combinaciones 1 y 2 , que poseen las mismas gluteninas de alto peso molecular $\left(2^{*}, 17+18,2+12\right)$. En la fracción rica en glutenina (50PI), no se encontró gran variación, y la mayor cantidad correspondió a las combinaciones 3 y 5 , con valores estadísticamente similares a la combinación 4, 6 y 7. Estos resultados sugieren que para mejorar la cantidad de proteínas del gluten, lo ideal sería encontrar genotipos con las combinaciones 2*,17+18, 5+10/Glu-A3c, Glu-B3g, Glu-D3b y 1, 17+18, 5+10/Glu-A3e, Glu-B3g, Glu-D3b. En la relación 50PS/50PI, la combinación con valores más altos fue $2^{*}, 17+18,2+12 / G l u-A 3 \mathrm{e}$, Glu-B3g,
Glu-D3b, seguida por la combinación $2 *, 17+18,2+12 /$ Glu-A3e, Glu-B3h, Glu-D3b.

En el Cuadro 4, se presentan las medias de los genotipos de la cruza Rebeca F2000 × Verano S91 en cada una de las variaciones alélicas de gluteninas de alto peso molecular de los loci Glu-A1 y Glu-D1, en las cuales para tiempo de amasado y fuerza de la masa el alelo 1 del locus $G l u-A 1$ y el $5+10$ del Glu-D1 presentaron los mayores valores de manera individual. En Glu-A1, no existieron diferencias entre las dos subunidades alélicas para alveograma-P/L; sin embargo, en Glu-Dl, el mayor valor fue para la subunidad 5+10, lo que implica una menor extensibilidad. Se encontró que los dos alelos de $G l u-A 1$ no presentaron diferencias en la fracción rica en gliadinas, pero en $G l u-D 1$, la mayor cantidad de gliadinas correspondió a los genotipos con el alelo $2+12$. Para la fracción rica en glutenina, los alelos del locus Glu-A1 no fue significativo; sin embargo, para el locus $G l u-D 1$, la mayor cantidad de gluteninas correspondió a los genotipos con el alelo 5+10.

En la relación gliadina/glutenina, en Glu-A1, no hubo diferencias entre los alelos 2 * y 1 ; sin embargo, en Glu-D1 se detectó una mayor proporción gliadina/glutenina con el alelo 2+12. Los genotipos con el alelo 5+10 mostraron una alta proporción de polímeros no extraíbles (50PI), en comparación con el $2+12$, lo que indica que los alelos individuales de gluteninas de alto peso molecular no son igualmente eficaces para polimerizar (Juhász et al., 2000). Las diferencias intrínsecas en los alelos y el diferente comportamiento durante la polimerización son responsables por la distribución del peso. Se sabe que a pesar de que los alelos de gluteninas de alto peso molecular son similares en su estructura, el alelo

Cuadro 3. Medias por combinación de alelos de gluteninas de alto (G-APM) y bajo (G-BPM) peso molecular de trigos harineros ${ }^{(1)}$.

\begin{tabular}{|c|c|c|c|c|c|c|c|c|}
\hline Combinación & 1(Verano S91) & 2 & 3 & 4 & 5 & 6 & 7 (Rebeca F2000) & $\mathrm{DSH}$ \\
\hline$\overline{\text { G-APM }}$ & $2^{*}, 17+18,2+12$ & $2 *, 17+18,2+12$ & $2 *, 17+18,5+10$ & $1,17+18,5+10$ & $1,17+18,5+10$ & $1,17+18,5+10$ & $1,17+18,5+10$ & - \\
\hline G-BPM & $\mathrm{e}, \mathrm{h}, \mathrm{b}$ & $\mathrm{e}, \mathrm{g}, \mathrm{b}$ & $\mathrm{c}, \mathrm{g}, \mathrm{b}$ & $\mathrm{e}, \mathrm{h}, \mathrm{b}$ & $\mathrm{e}, \mathrm{g}, \mathrm{b}$ & $\mathrm{c}, \mathrm{h}, \mathrm{b}$ & $\mathrm{c}, \mathrm{g}, \mathrm{b}$ & - \\
\hline $\mathrm{N}$ & 18 & 50 & 38 & 28 & 29 & 32 & 35 & - \\
\hline MTA & $2,32 \mathrm{c}$ & $2,20 \mathrm{c}$ & $3,50 \mathrm{a}$ & $2,83 b$ & $3,11 \mathrm{ab}$ & $3,51 \mathrm{a}$ & $3,42 \mathrm{a}$ & 0,66 \\
\hline ALVW & $201,47 b$ & $212,38 b$ & $378,74 a$ & $367,88 \mathrm{a}$ & $329,04 \mathrm{a}$ & $345,41 \mathrm{a}$ & $353,63 \mathrm{a}$ & 82,00 \\
\hline ALVPL & $0,606 \mathrm{~d}$ & $0,850 \mathrm{bcd}$ & $1,362 \mathrm{a}$ & $0,845 \mathrm{bcd}$ & $1,080 \mathrm{ab}$ & $0,731 \mathrm{~cd}$ & $0,965 b c$ & 0,38 \\
\hline 50PS (\%) & $66,29 a$ & $66,45 \mathrm{a}$ & $63,09 \mathrm{~b}$ & $65,09 \mathrm{ab}$ & $63,38 b$ & $65,03 \mathrm{ab}$ & $64,52 \mathrm{ab}$ & 20,40 \\
\hline 50PI (\%) & $33,70 \mathrm{~b}$ & $33,54 b$ & $36,90 \mathrm{a}$ & $34,90 \mathrm{ab}$ & $36,61 \mathrm{a}$ & $34,96 \mathrm{ab}$ & $35,47 \mathrm{ab}$ & 17,20 \\
\hline 50PS/50PI & $1,96 \mathrm{ab}$ & $1,99 \mathrm{a}$ & $1,70 \mathrm{c}$ & $1,86 \mathrm{abc}$ & $1,73 \mathrm{bc}$ & $1,86 a b c$ & $1,81 \mathrm{abc}$ & 0,23 \\
\hline
\end{tabular}

${ }^{(1)}$ Medias con la misma letra dentro de hileras no son estadísticamente diferentes por la prueba de Tukey al 5\% de probabilidad. MTA, tiempo de amasado (min); ALVW, fuerza de la masa $\left(10^{-4} \mathrm{~J}\right)$; ALVPL, extensibilidad de la masa; 50PS, fracción rica en gliadina; 50PI, fracción rica en glutenina; 50PS+50PI, proteína total de reserva; 50PS/50PI, relación fracción rica en gliadina/fracción rica en glutenina; DSH, diferencia significativa honesta. 
5+10 tiene un residuo de cisteína adicional, lo que está relacionado con su mayor peso molecular (Southan y MacRitchie, 1999).

En el Cuadro 5, se muestran las medias de las líneas de la cruza Rebeca F2000 × Verano S91, en cada una de las variaciones alélicas de gluteninas de bajo peso molecular de los loci Glu-A3 y Glu-B3. Para tiempo de amasado y fuerza de la masa, el alelo Glu-A3c tuvo los mayores valores, lo que confiere calidad panadera a los genotipos con dicho alelo; en Glu-B3, no hubo diferencias entre los dos alelos. La mejor extensibilidad de la masa correspondió al alelo GluA3e y Glu-B3h, con menores valores de alveograma-P/L. En la relación 50PS/50PI, el alelo Glu-A3e presentó un mayor valor que en $G l u-A 3 c$, lo que se debe a un mayor contenido de la fracción rica en gliadina que en la fracción rica en glutenina.

En el Cuadro 6, se muestra la comparación de medias de los ambientes para cada una de las variables de calidad, en las fracciones de proteínas y en la relación de ellas. El tiempo de amasado fue mucho mayor con el aumento de la temperatura ambiental en las fechas de siembra tardías. El alveograma-W presentó la misma fuerza de la masa en todos los ambientes, pues su control es principalmente genotípico. En el alveograma-P/L, el menor valor fue para el tratamiento control; por lo tanto, el aplicar azufre o sembrar tarde no implica que se produzca mayor extensibilidad de la masa. La fracción rica en gliadina fue mayor con aplicación de azufre, y menor con alto calor, lo que coincide con los resultados de Tea et al. (2004), quienes encontraron que las fertilizaciones con $\mathrm{N}$ y $\mathrm{S}$, aplicadas en antesis, juegan un papel importante en la síntesis de proteínas de almacenamiento, su grado de polimerización y en las propiedades mixográficas de la masa. El incremento en la fracción rica en gliadinas, en el ambiente normal (tratamiento 4), se explica en gran parte por la aplicación de una dosis adecuada de nitrógeno, pues este nutriente aumenta la cantidad total de proteína del grano de trigo, traduciéndose en un incremento en la cantidad de gliadinas y gluteninas (Wieser y Seilmeier, 1998; Triboï et al., 2000).

Para la fracción rica en glutenina, el mayor valor se obtuvo en calor y riego limitado, probablemente relacionado con un llenado de grano menor que en condiciones normales, lo que corrobora los resultados

Cuadro 4. Medias por alelo de las gluteninas de alto peso molecular (loci $A 1$ y D1), en líneas de la cruza Rebeca F2000 $\times$ Verano S91 de trigos harineros ${ }^{(1)}$.

\begin{tabular}{|c|c|c|c|c|c|c|}
\hline \multirow[t]{2}{*}{ Variable } & \multicolumn{3}{|c|}{ Locus $G l u-A 1$} & \multicolumn{3}{|c|}{ Locus Glu-DI } \\
\hline & 1 & $2 *$ & DMS & $2+12$ & $5+10$ & $\mathrm{DSH}$ \\
\hline Tiempo de amasado (min) & $3,21 \mathrm{a}$ & $2,68 b$ & 0,19 & $2,23 b$ & $3,28 \mathrm{a}$ & 0,18 \\
\hline Alveograma-W $\left(10^{-4} \mathrm{~J}\right)$ & $352,33 a$ & $268,97 b$ & 24,70 & $209,8 b$ & $358,29 a$ & 21,30 \\
\hline Alveograma-P/L $(0,1-6)$ & $0,906 \mathrm{a}$ & $0,993 \mathrm{a}$ & 0,11 & $0,793 b$ & $1,009 \mathrm{a}$ & 0,11 \\
\hline $50 \mathrm{PS}(\%)$ & $64,52 \mathrm{a}$ & $65,23 \mathrm{a}$ & 7,68 & $66,41 \mathrm{a}$ & $64,20 \mathrm{~b}$ & 8,20 \\
\hline 50PI (\%) & $35,47 \mathrm{a}$ & $34,76 \mathrm{a}$ & 6,08 & $33,58 b$ & $35,80 \mathrm{a}$ & 6,60 \\
\hline 50PS/50PI & $1,81 \mathrm{a}$ & $1,87 \mathrm{a}$ & 0,08 & $1,97 \mathrm{a}$ & $1,79 b$ & 0,08 \\
\hline
\end{tabular}

${ }^{(1)}$ Medias con misma letra dentro de hileras son estadísticamente iguales, por la prueba de Tukey al 5\% de probabilidad. 50PS, fracción rica en gliadina; 50PI, fracción rica en glutenina; 50PS/50PI, relación fracción rica en gliadina/ fracción rica en glutenina; DSH, diferencia significativa honesta.

Cuadro 5. Medias por alelo de las gluteninas de bajo peso molecular (loci Glu-A3 y Glu-B3), en la cruza Rebeca F2000 x Verano S91 de trigos harineros ${ }^{(1)}$.

\begin{tabular}{|c|c|c|c|c|c|c|}
\hline \multirow[t]{2}{*}{ Variable } & \multicolumn{3}{|c|}{ Locus $G l u-A 3$} & \multicolumn{3}{|c|}{ Locus $G l u-B 3$} \\
\hline & $\mathrm{c}$ & e & DMS & $\mathrm{h}$ & g & DSH \\
\hline Tiempo de amasado (min) & $3,44 \mathrm{a}$ & $2,57 \mathrm{~b}$ & 0,17 & $2,99 \mathrm{a}$ & $2,96 \mathrm{a}$ & 0,80 \\
\hline Alveograma-W $\left(10^{-4} \mathrm{~J}\right)$ & $364,01 \mathrm{a}$ & $270,79 b$ & 24,10 & $322,59 a$ & $310,55 \mathrm{a}$ & 101,30 \\
\hline Alveograma-P/L $(0,1-6)$ & $1,027 \mathrm{a}$ & $0,87 \mathrm{~b}$ & 0,11 & $0,74 \mathrm{~b}$ & $1,04 \mathrm{a}$ & 0,39 \\
\hline $50 \mathrm{PS}(\%)$ & $64,17 \mathrm{a}$ & $65,42 \mathrm{a}$ & 7,56 & $65,34 \mathrm{a}$ & $64,60 \mathrm{a}$ & 7,86 \\
\hline $50 \mathrm{PI}(\%)$ & $35,82 \mathrm{a}$ & $34,57 \mathrm{a}$ & 6,25 & $34,66 \mathrm{a}$ & $35,39 a$ & 6,56 \\
\hline 50PS/50PI & $1,79 b$ & $1,89 \mathrm{a}$ & 0,08 & $1,88 \mathrm{a}$ & $1,82 \mathrm{a}$ & 0,09 \\
\hline
\end{tabular}

${ }^{(1)}$ Medias con misma letra dentro de hileras son estadísticamente iguales, por la prueba de Tukey al 5\% de probabilidad. 50PS, fracción rica en gliadina; 50PI, fracción rica en glutenina; 50PS/50PI, relación fracción rica en gliadina/ fracción rica en glutenina. DSH, diferencia significativa honesta. 
Cuadro 6. Medias por ambiente (tratamientos aplicados) de líneas de trigos harineros de la cruza Rebeca F2000 × Verano S91 ${ }^{(1)}$.

\begin{tabular}{|c|c|c|c|c|c|c|}
\hline Variable & 1 & 2 & 3 & 4 & 5 & DSH \\
\hline $\mathrm{N}$ & 46 & 48 & 44 & 50 & 46 & \\
\hline Tiempo de amasado (min) & $2,98 \mathrm{~b}$ & $2,57 \mathrm{~d}$ & $2,77 \mathrm{c}$ & $2,94 b c$ & $3,62 \mathrm{a}$ & 0,17 \\
\hline Alveograma-W $\left(10^{-4} \mathrm{~J}\right)$ & $331,47 \mathrm{a}$ & $308,64 a$ & $308,22 \mathrm{a}$ & $307,92 \mathrm{a}$ & $318,50 \mathrm{a}$ & 28,50 \\
\hline Alveograma-P/L & $1,09 \mathrm{a}$ & $0,95 \mathrm{~b}$ & $0,98 \mathrm{ab}$ & $0,69 \mathrm{c}$ & $1,06 \mathrm{ab}$ & 0,14 \\
\hline $50 \mathrm{PS}(\%)$ & $62,91 \mathrm{c}$ & $69,34 \mathrm{a}$ & $65,57 \mathrm{~b}$ & $66,49 b$ & $59,61 \mathrm{~d}$ & 10,20 \\
\hline 50PI (\%) & $37,08 \mathrm{~b}$ & $30,65 \mathrm{~d}$ & $34,42 \mathrm{c}$ & $33,50 \mathrm{c}$ & $40,38 \mathrm{a}$ & 10,10 \\
\hline $50 \mathrm{PS} / 50 \mathrm{PI}$ & $1,69 \mathrm{c}$ & $2,26 \mathrm{a}$ & $1,90 \mathrm{~b}$ & $1,98 \mathrm{~b}$ & $1,47 \mathrm{~d}$ & 0,14 \\
\hline
\end{tabular}

${ }^{(1)}$ Medias con misma letra dentro de hileras son estadísticamente iguales, por la prueba de Tukey al 5\% de probabilidad. 50PS, fracción rica en gliadina; 50PI, fracción rica en glutenina; 50PS/50PI, relación fracción rica en gliadina/ fracción rica en glutenina; DSH, diferencia significativa honesta.

de Guttierri et al. (2001), quienes al aplicar déficits severos de agua, afectaron la calidad final de diversas cultivares de trigo; de igual manera, Labuschagne et al. (2009) observaron que al incrementar las temperaturas durante el llenado de grano se aumenta la cantidad de fracciones de gluteninas. Daniel \& Triboï (2002) al analizar la cinética de acumulación de gliadinas y gluteninas de alto y bajo peso molecular, en cultivos con y sin riego, no encontraron un efecto significativo de la sequía, concluyendo que la sequía en post-antesis acorta el periodo de llenado de grano, antes que se lleve a cabo la insolubilización de polímeros. Para la relación 50PS/50PI, el mayor valor correspondió a la fertilización con azufre, resultados similares a los encontrados por Tea et al. (2004).

\section{Conclusiones}

1. El genotipo y el ambiente tienen gran efecto sobre las variables de calidad de la masa, así como las fracciones de glutenina y gliadina, y su relación.

2. El tiempo de amasado es mayor cuando se aumenta la temperatura, y la mejor extensibilidad de la masa se obtiene en el ambiente bajo condiciones normales.

3. La fracción rica en gliadina y la relación 50PS/50PI son mayores cuando se realiza la fertilización con azufre, y se obtiene incremento de la fracción rica en glutenina con riego limitado y aumento de temperaturas durante el llenado de grano.

\section{Agradecimientos}

Al Consejo Nacional de Ciencia y Tecnología y al Instituto Nacional de Investigaciones Forestales, Agrícolas y Pecuarias, por el financiamiento otorgado para la realización de la presente investigación.

\section{Referencias}

AACC INTERNATIONAL. Approved methods of the AACC International. $9^{\text {th }}$ ed. St. Paul: AACC International, 1995.

BRANLARD, G.; DARDEVET, M.; AMIOUR, N.; IGREJAS, G. Allelic diversity of HMW and LMW glutenin subunits and omega-gliadins in French bread wheat (Triticum aestivum L.). Genetic Resources and Crop Evolution, v.50, p.669-679, 2003.

DANIEL, C.; TRIBOÏ, E. Changes in wheat protein aggregation during grain development: effects of temperatures and water stress. European Journal of Agronomy, v.16, p.1-12, 2002.

DANIEL, C.; TRIBOÏ, E. Effects of temperature and nitrogen nutrition on the grain composition of winter wheat: effects on gliadin content and composition. Journal of Cereal Science, v.32, p.45-56, 2000.

DUPONT, F.M.; ALTENBACH, S.B. Molecular and biochemical impacts of environmental factors on wheat grain development and protein synthesis. Journal of Cereal Science, v.38, p.133-146, 2003.

ESPITIA, R.E.; PEÑA, B.R.J.; VILLASEÑOR, M.H.E.; HUERTA, E.J.; LIMÓN, O.A. Calidad industrial de trigos harineros mexicanos para temporal. I. Comparación de variedades y causas de la variación. Revista Fitotecnia Mexicana, v.26, p.249-256, 2003.

GRAYBOSCH, R.A.; PETERSON, C.J.; BAEZINGER, P.S.; SHELTON, D.R. Environmental modification of hard red winter wheat flour protein composition. Journal of Cereal Science, v.22, p.45-51, 1995.

GUTTIERI, M.J.; STARK, J.C.; O'BRIEN, K.; SOUZA, E. Relative sensitivity of spring wheat grain yield and quality parameters to moisture deficit. Crop Science, v.41, p.327-335, 2001.

JACKSON, E.A.; MOREL, M.H.; SONTAG-STROHM, T.; BRANLARD, G.; METAKOVSKY, E.V.; REDAELLI, R. Proposal for combining the classification systems of alleles of Gli-1 and Glu-3 loci in bread wheat (Triticum aestivum L.). Journal of Genetics and Breeding, v.50, p.321-336, 1996.

JUHÁSZ, A.; BÉKÉS, F.; VIDA, G.Y.; LÁNG, L.; TAMÁS, L.; BEDÖ, Z. Quantitative analyses of storage proteins of an old Hungarian wheat population using the SE-HPLC method. In: INTERNATIONAL WOKSHOP on GLUTEN, 7., 2000, Bristol. Proceedings. Cambridge: Royal Society of Chemistry, 2000. p.34-37. 
LABUSCHAGNE, M.T.; ELAGO, O.; KOEN, E. Influence of extreme temperatures during grain filling on protein fractions, and its relationship to some quality characteristics in bread, biscuit, and durum wheat. Cereal Chemistry, v.86, p.61-66, 2009.

PANOZZO, J.F.; EAGLES, H.A. Cultivar and environment effects on quality characters in wheat. II. Protein. Australian Journal of Agricultural Research, v.51, p.629-636, 2000.

PAYNE, P.I.; SEEKINGS, J.A.; WORLAND, A.J.; JARVIS, M.G.; HOLT, L.M. Allelic variation of glutenin subunits and gliadins and its effect on breadmaking quality in wheat: analysis of $\mathrm{F}_{5}$ progeny from "Chinese Spring" $x$ "Chinese Spring" (Hope 1A). Journal of Cereal Science, v.6, p.103-118, 1987.

PEÑA, R.J.; AMAYA, A.; RAJARAM, S.; MUJEEB-KAZI, A. Variation in quality characteristics associated with some spring 1B/1R translocation wheats. Journal of Cereal Science, v.12, p.105-112, 1990 .

PEÑA, R.J.; GONZÁLEZ, H.S.; CERVANTES, F. Relationship between Glu-D1/GluB3 allelic combinations and breadmaking quality-related parameters commonly used in wheat breeding. In: GLUTEN WORKSHOP, 8., 2004, Viterbo. Proceedings. Cambridge: Royal Society of Chemistry, 2004. p.156-157.

RADOVANOVIC, C.N.; CLOUTIER, S.; BROWN, D.; HUMPHREYS, D.G.; LUKOW, O.M. Genetic variance for gluten strength contributed by high molecular weight glutenin proteins. Cereal Chemistry, v.79, p.843-849, 2002.

SAS INSTITUTE. SAS/STAT software: changes and enhancements through. Release 6.12. Cary: SAS Institute, 1997.

SHEWRY, P.R.; HALFORD, N.G. Cereal seed storage proteins: structures, properties and role in grain utilization. Journal of Experimental Botany, v.53, p.947-958, 2002.

SHEWRY, P.R.; HALFORD, N.G.; TATHAM, A.S.; POPINEAU, Y.; LAFIANDRA, D.; BELTON, P.S. The high molecular weight subunits of wheat glutenin and their role in determining wheat processing properties. Advances in Food and Nutrition Research, v.45, p.219-302, 2003.
SINGH, N.K.; SHEPHERD, K.W.; CORNISH, G.B. A simplified SDS-PAGE procedure for separating LMW subunits of glutenin. Journal of Cereal Science, v.14, p.203-208, 1991.

SOUTHAN, M.; MACRITCHIE, F. Molecular weight distribution of wheat proteins. Cereal Chemistry, v.76, p.827-836, 1999.

SOUZA, E.J.; MARTIN, J.M.; GUTTIERI, M.J.; O'BRIEN, K.M.; HABERNICHT, D.K.; LANNING, S.P.; MCLEAN, R.; CARLSON, G.R.; TALBERT, L.E. Influence of genotype, environment, and nitrogen management on spring wheat quality. Crop Science, v.44, p.425-432, 2004.

SUCHY, J.; LUKOW, O.M.; BROWN, D.; DEPAUW, R.; FOX, S.; HUMPHREYS, S. Rapid assessment of glutenin and gliadin in wheat by UV spectrophotometer. Crop Science, v.47, p.91-99, 2007.

TEA,I.; GENTER, T.; NAULET, N.;BOYER, V.; LUMMERZHEIM, M.; KLEIBER, D. Effect of foliar sulfur and nitrogen fertilization on wheat storage protein composition and dough mixing properties. Cereal Chemistry, v.81, p.759-766, 2004.

TRIBOÏ, E.; ABAD, A.; MICHELENA, A.; LLOVERAS, J.; OLLIER, J.L.; DANIEL, C. Environmental effects on the quality of two wheat genotypes. 1.Quantitative and qualitative variation of storage proteins. European Journal of Agronomy, v.13, p.47-64, 2000.

UTHAYAKUMARAN, S.; LUKOW, O.M. Improving wheat for bread and tortilla production by manipulating glutenin-to-gliadin ratio. Journal of the Science of Food and Agriculture, v.85, p.2111-2118, 2005.

WIESER, H.; SEILMEIER, W. The influence of nitrogen fertilization on quantities and proportions of different protein types in wheat flour. Journal of the Science of Food and Agriculture, v.76, p.49-55, 1998.

ZHU, J.; KHAN, K. Effects of genotype and environment on glutenin polymers and breadmaking quality. Cereal Chemistry, v.78, p.125-130, 2001.

Recibido el 20 de junio de 2010 y aprobado el 27 de agosto de 2010

Pesq. agropec. bras., Brasília, v.45, n.9, p.989-996, set. 2010 